\title{
EL DESARROLLO DEL SECTOR ASEGURADOR Y EL CRECIMIENTO ECONÓMICO: UN ANÁLISIS EMPÍRICO PARA 47 PAÍSES ${ }^{1}$
}

\author{
DEVELOPMENT OF THE INSURANCE SECTOR AND ECONOMIC GROWTH: AN \\ EMPIRICAL ANALYSIS FOR 47 COUNTRIES
}

\section{Anelena Sabater Castro²}

\section{Resumen}

Este estudio examina el efecto del desarrollo del mercado asegurador -tanto del segmento de vida como de no vida ${ }^{3}$ - sobre el crecimiento económico, así como las condiciones (grado de desarrollo financiero, nivel de ingresos y entorno institucional) que afectan el nexo de ambas variables. Para ello se utiliza el método generalizado de momentos (GMM), con un modelo dinámico de datos de panel para 47 países, entre 1990 y 2014. Los resultados revelan que existe un impacto positivo del desarrollo del mercado de seguros sobre el crecimiento económico. Además, la relación entre el desarrollo del mercado asegurador y el crecimiento económico se ve aminorada en un entorno institucional relativamente más sólido. Por otra parte, los resultados sugieren que el impacto positivo del desarrollo del mercado de seguros en el crecimiento económico se mitiga en los países de ingresos altos.

Palabras clave: desarrollo del mercado asegurador; crecimiento económico; modelo dinámico de datos de panel

Doi: $\underline{\text { http://dx.doi.org/10.15359/eys.22-52.5 }}$

Fecha de recepción: 04-08-2017. Fechas de reenvíos: 17-11-2017, 22-11-2017, 27-11-2017. Aceptado el 01-122018. Publicado el 11-12-2017.

\footnotetext{
${ }^{1}$ Este trabajo es una adaptación del proyecto de graduación para optar por el grado de Máster en Finanzas, Banca y Seguros, de la Universidad de Málaga, España.

${ }^{2}$ Máster en Finanzas, Banca y Seguros de la Universidad de Málaga (España), Bachiller en Economía y Bachiller en Dirección de Empresas de la Universidad de Costa Rica. Analista de Inteligencia de Negocios en InterClear Central de Valores, Costa Rica. Correo electrónico: asabaterc@uma.es

${ }^{3}$ Existen dos grandes categorías de seguros: vida y no vida. Los seguros de vida se caracterizan porque el pago de la cantidad pactada (suma asegurada) depende del fallecimiento o supervivencia del asegurado. Los seguros generales o de no vida cubren riesgos que puedan ocasionar daños a los bienes o derechos del asegurado, etc.
} 88

Anelena Sabater Castro

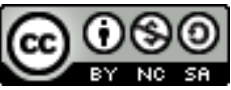

Revista Economía y Sociedad by Universidad Nacional is licensed under a CreativeCommons Reconocimiento-NoComercial- 


\section{Abstract}

This paper studies the effect of the development of the life and nonlife insurance market on economic growth, as well as the conditions (degree of financial development, income and institutional environment) that affect the relationship between both variables. The Generalized Method of Moments (GMM) was used, with a dynamic panel data model for 47 countries between 1990 and 2014 . Results indicate that there is a positive impact of the development of the insurance market on economic growth. In addition, the relationship between the insurance market and economic growth decreases in a relatively sounder institutional environment. On the other hand, the positive impact of the development of the insurance market on economic growth is mitigated in high-income countries.

Keywords: development of the insurance market; economic growth; dynamic panel data model

\section{Introducción}

"El desarrollo del sector asegurador desempeña un papel trascendental en el desarrollo financiero y económico de un país" (Feyen, Lester y Rocha, 2011, p. 34). La afirmación anterior es habitual en estudios que tratan sobre el desarrollo del mercado asegurador y su vínculo con el crecimiento económico, debido a que teóricamente no es difuso y hay un consenso generalizado: un mercado asegurador desarrollado contribuye significativamente al crecimiento económico.

En términos generales las aseguradoras cumplen dos funciones principales: una de transferencia de riesgos y otra de índole financiera. La primera función hace referencia a la administración de riesgos y se refiere a la reducción de la incertidumbre (ya sea proveniente de realizar diversas actividades en la economía o la relacionada con situaciones adversas a nivel personal). En el caso de los seguros de no vida, por ejemplo, el seguro facilita el comercio internacional, ya que permite administrar los riesgos que conlleva el exportar o importar bienes. En términos de seguros de vida, esta función permite el traspaso de los riesgos del asegurado al asegurador ${ }^{4}$, liberando de esta forma al primero de la preocupación de poner en peligro su economía o la de sus allegados, en casos como la muerte prematura o jubilación.

Por otro lado, diversos autores (Herce, Delgado, Azpeitia, Blasco y Hernández 2013; Chamberlain, Coetze y Camargo 2017) vinculan esta función con el fomento del

\footnotetext{
${ }^{4} \mathrm{El}$ asegurado se refiere a la persona a quien protege el seguro; por su parte, el asegurador hace referencia a la entidad aseguradora.
} 
emprendimiento, debido a que algunos riesgos inherentes a determinadas actividades pueden ser cubiertos por medio de un seguro ${ }^{5}$. Ejemplo de ello son las conclusiones a las que llegan Dercon y Christiaensen (2007), quienes afirman que la ausencia de seguros causa ineficiencia en las decisiones de producción de los agricultores de Etiopía, ya que estos últimos tienden a participar en actividades de bajo rendimiento ( $y$, por ende, de bajo riesgo) por la ausencia de un mercado asegurador profundo.

En cuanto a la segunda función, de carácter financiero, esta tiene diversos matices. Primeramente, tal como expone Barr (2010), el seguro va en consonancia con la adecuada regulación funcional del ahorro y, en contraposición con la autofinanciación (una alternativa del seguro), es una solución superior, de mayor bienestar y evita el exceso de ahorro. Por otro lado, las compañías aseguradoras son importantes inversores institucionales y contribuyen al desarrollo del mercado de capitales. Numéricamente los datos son brutales, según Insurance Europe, la inversión agregada de las compañías de seguros europeas representó el 60,58\% de su Producto Interno Bruto (PIB) en el año 2015.

Concretamente y de forma sintética, siguiendo a Focarelli (2017) y Chamberlain, Coetze y Camargo (2017), se puede afirmar que las aseguradoras 1) reducen la incertidumbre y volatilidad, 2) promueven estabilidad financiera a través de inversiones a largo plazo y 3) fomentan las buenas prácticas de ahorro.

Además, Herce et al. (2013) ponen de manifiesto que el sector asegurador no solo influye en el crecimiento económico, sino que existe una relación bidireccional, en la cual el impulso económico influye en la contratación de pólizas de seguros y el desarrollo del sector asegurador contribuye positivamente al desarrollo económico. Países como España y Alemania son ejemplos claros de lo anterior, lo cual se puede observar en las Figuras $\underline{1}$ y $\underline{2}$, donde se muestra la evolución del volumen de primas y el PIB para estas dos economías desarrolladas.

${ }^{5}$ Por ejemplo, los microseguros fungen como un mecanismo de protección contra el riesgo dirigido a personas de bajos ingresos.

90

Anelena Sabater Castro

cc) (i) (2)

Revista Economía y Sociedad by Universidad Nacional is licensed under a CreativeCommons Reconocimiento-NoComercial- 


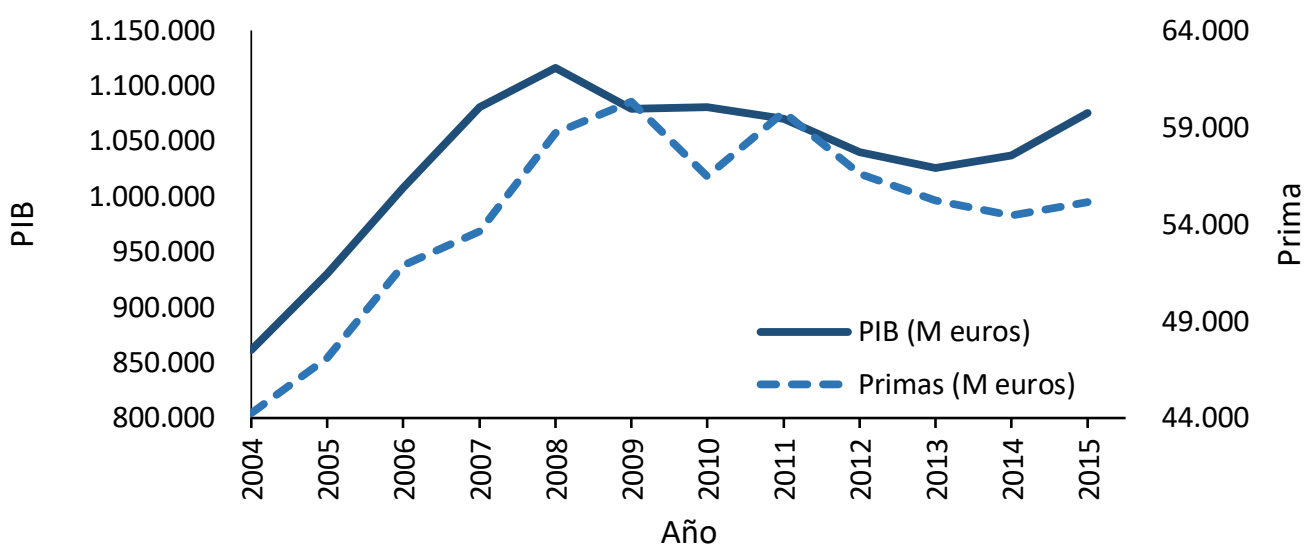

Figura 1. España: Evolución PIB y Primas, 2004-2015. Fuente: elaboración a partir de la información publicada por Insurance Europe (2016) y OCDE (2016).

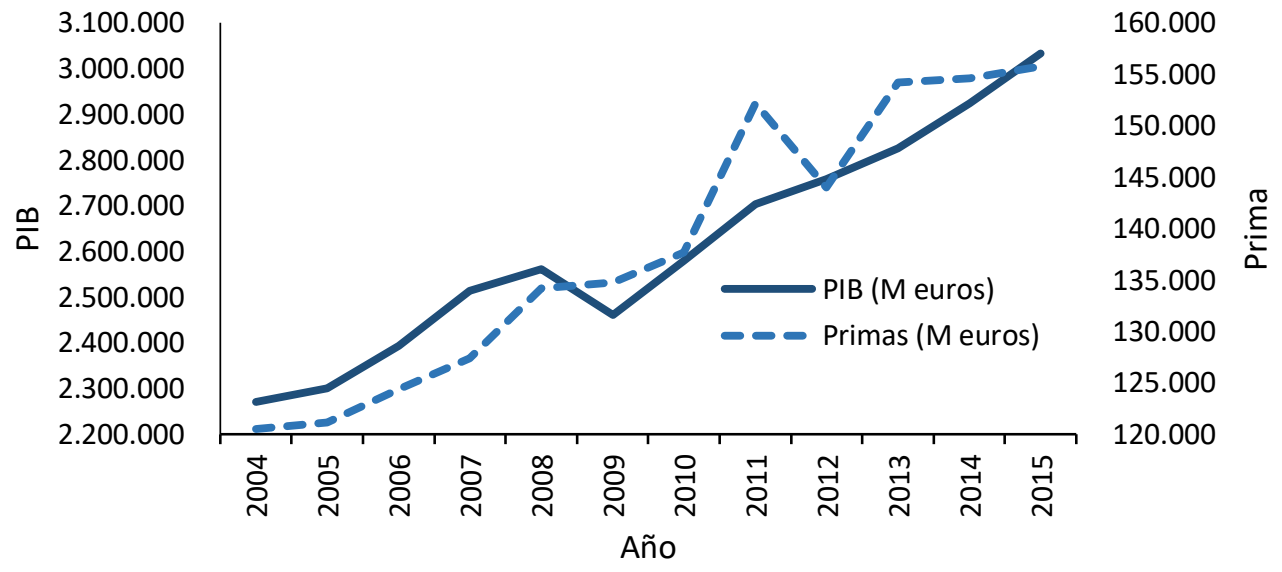

Figura 2. Alemania: Evolución PIB y Primas, 2004-2015. Fuente: elaboración a partir de la información publicada por Insurance Europe (2016) y OCDE (2016).

Debido a la importancia del mercado asegurador en una economía, diversas investigaciones han puesto de manifiesto la necesidad de más estudios que busquen analizar el vínculo entre el desarrollo del mercado asegurador y el crecimiento económico (al respecto, véase Haiss y Sumegi, 2008; Outreville, 2013; Focarelli, 2017). Lo anterior se debe a que en su mayoría la literatura empírica se basa en analizar únicamente el efecto del desarrollo de los seguros del segmento de vida - sin abarcar no vida-. Además, hay una necesidad latente de estudios que analicen el efecto de diversas variables de otra índole (por ejemplo, el rol institucional) en la relación entre el desarrollo asegurador y el crecimiento económico. De manera tal que se analice si, y qué medida, diversas variables condicionan (refuerzan o mitigan) la relación entre el desarrollo del sector asegurador y el crecimiento económico. 
Ante ello, se establece como objetivo del presente estudio analizar el efecto de la actividad aseguradora (considerando el segmento de vida y no vida) sobre el crecimiento económico, por medio de un panel dinámico con datos de 1990 a 2014 para 47 países. El principal aporte que supone esta investigación radica en avanzar en la explicación empírica del efecto del desarrollo del mercado asegurador en el crecimiento económico, generando evidencia econométrica con datos recientes. Además, se incorporan en el estudio variables financieras e institucionales.

\section{Metodología}

\subsection{Especificación del modelo}

Con el propósito de evaluar el efecto del mercado asegurador en el crecimiento económico y siguiendo a Arena (2008) y a Cheen et al. (2012) se parte de la siguiente regresión dinámica a estimar:

$$
Y_{i t}=\alpha Y_{\text {inicial }}+\beta I N S U_{i t}+\delta^{\prime} X_{i t}+\mu_{i}+\varepsilon_{i t}
$$

donde:

$Y_{i t}$ : crecimiento económico para el país i en el periodo $t$

$Y_{i \text { inicial }}$ : valor inicial del crecimiento económico para el país i

$X_{i t}$ : matriz de variables explicativas de control para el país i en el periodo $t$

$I N S U_{i t}$ : se refiere a una de las variables proxies del desarrollo del mercado de seguros (ratio de penetración de seguros ${ }^{6}$ de vida, no vida y total) para el país i en el periodo $t$

$\beta$ : vector de coeficientes estimados de la variable que representa el desarrollo del mercado asegurador

$\delta^{\prime}$ : vector de coeficientes estimados de las variables de control

$\mu_{i}$ : efecto inobservable del país i

$\varepsilon_{i t}$ : término de perturbación aleatoria

Debido a que existe una relación entre la variable dependiente y las explicativas de manera bidireccional y, a su vez, se puede dar una relación de dependencia entre las variables explicativas, se realiza un análisis para datos de panel a través de modelos econométricos dinámicos. Más específicamente, se aplica el método generalizado de momentos (MGM) y concretamente el estimador desarrollado por Arellano y Bond (1991), Arellano y Bover (1995) y Blundella y Bond (1998), el cual permite lidiar con paneles dinámicos.

Esta metodología permite controlar problemas de endogeneidad en las variables explicativas, los efectos no observados de tiempo y país, así como heterocedasticidad y autocorrelación dentro de los datos. Además, permite contar con la variable dependiente en función de su

${ }^{6}$ Se refiere a Primas/PIB y es la medida más comúnmente usada para evaluar la importancia del seguro para la economía (Outreville, 1998).

92

Anelena Sabater Castro

(c) (1) (2)

Revista Economía y Sociedad by Universidad Nacional is licensed under a CreativeCommons Reconocimiento-NoComercial- 
propio pasado; es decir, rezagada en el tiempo como variable explicativa. No obstante, en este caso se considera el valor inicial del crecimiento económico, tal como se puede observar en la ecuación $(1)^{7}$. Cabe destacar que el GMM es una metodología ampliamente utilizada para estudiar el efecto del desarrollo del sector financiero sobre el crecimiento económico ${ }^{8}$.

Es posible aplicar primeras diferencias a la ecuación (1) y reescribirla de la siguiente forma:

$$
\Delta Y_{i t}=\alpha \Delta Y_{i \text { inicial }}+\beta \Delta I N S U_{i t}+\delta^{\prime} \Delta X_{i t}+\Delta \varepsilon_{i t}
$$

De forma tal que se elimina el efecto específico de cada país i.

Esta metodología implica el uso de variables instrumentales para mejorar la eficiencia del modelo, lo cual permite: 1) tratar la endogeneidad de las variables explicativas y 2) lidiar con el problema de que el error $\left(\Delta \varepsilon_{i t}\right)$ esté correlacionado con la primera diferencia del rezago de la variable dependiente $\left(\Delta y_{i t-1}\right)$.

Con el fin de reducir los posibles sesgos y la imprecisión asociados con los estimadores, se utiliza System GMM en dos etapas, cuyo comando en el paquete estadístico STATA es "xtabond2", desarrollado por Roodman (2009). Este método, además de utilizar las diferencias de los retardos, también emplea retardos en niveles de las variables como instrumentos.

Además, se estima un modelo extendido, el cual contempla variables de interacción, que representan el producto de las dos variables (una representa el desarrollo del sector asegurador) y se refieren a la consideración conjunta de ambas variables. En este caso, siguiendo a Chen, Lee y Lee (2012), el modelo es especificado de la siguiente forma:

$$
\Delta Y_{i t}=\alpha \Delta Y_{\text {inicial }}+\beta \Delta I N S U_{i t}+\gamma \Delta\left[I N S U_{i t} x V_{i t}\right]+\rho \Delta V_{i t}+\delta^{\prime} \Delta X_{i t}+\Delta \varepsilon_{i t}
$$

Donde:

$V_{i t}$ : término con el que se realiza la interacción

$I N S U_{i t} x V_{i t}$ : se refiere al término de interacción

Al igual que Chen et al. (2012), se establecen las siguientes hipótesis:

\footnotetext{
${ }^{7}$ Al respecto, véase Arena (2008) quien también utiliza el valor inicial de la variable dependiente como variable explicativa.

${ }^{8}$ Para mayor detalle remítase a Levine, Loayza y Beck (2000).
} 
1. Si $\beta>0, \rho>0$ y $\gamma>0$ entonces el desarrollo de mercado de seguros (según se refiera al segmento de vida, no vida o ambos) tiene un efecto positivo en el crecimiento económico y el término $V_{i t}$ afecta este impacto positivamente. Además, $V_{i t}$ tendría un impacto positivo en la variable dependiente, en este caso el crecimiento económico.

2. Si $\beta>0, \rho>0$ y $\gamma<0$ entonces el desarrollo de mercado de seguros (según se refiera al segmento de vida, no vida o ambos) tiene un impacto positivo en el crecimiento económico. No obstante, la variable incluida en $V_{i t}$ está afectando este efecto de forma adversa; es decir, mitiga el impacto.

Cabe señalar que las pruebas de especificación consideradas fueron el test de Arellano-Bond, test de Sargan y test de Hansen. El test de Arellano-Bond permite analizar si existe autocorrelación de segundo orden en las primeras diferencias de los errores, una restricción importante del estimador es que no debe existir tal autocorrelación. Por su parte, el test de Sargan permite identificar la sobreidentificación de las ecuaciones. Por último, el test de Hansen se refiere a una prueba para la validez de los instrumentos.

\subsection{Datos}

Se utiliza un panel de datos para 47 países (véase el Apéndice 1) durante el periodo de 19902014, los datos se promedian en cada uno de los cinco intervalos de cinco años que componen el período de 1990 a 2014 (1990-1994; 1995-1999; 2000-2004; 2005-2009; 2010-2014), de modo tal que se tienen cinco observaciones de cada variable por país. Esto posibilita: 1) aminorar la influencia de los ciclos económicos y 2) utilizar el $\mathrm{GMM}^{9}$.

En cuanto a las variables incluidas en el análisis, estas se detallan en la Tabla $\underline{\underline{1}}$.

${ }^{9}$ Esta metodología es acorde al panel de datos, el cual está compuesto por una gran cantidad de países (N) y un número reducido de periodos de tiempo (T).

94

Anelena Sabater Castro 
Tabla 1.

Definición y fuente de las variables utilizadas

\begin{tabular}{|c|c|c|c|c|}
\hline Categoría & Variable & Nombre & Definición/construcción & Fuente \\
\hline \multirow{3}{*}{ Seguros } & $\begin{array}{l}\text { Ratio de penetración en } \\
\text { seguros de vida }\end{array}$ & LIP & $\begin{array}{l}\text { Primas de seguros de vida como porcentaje del } \\
\text { PIB. }\end{array}$ & $\begin{array}{l}\text { Swiss Reinsurance Company } \\
(\underline{2016)}\end{array}$ \\
\hline & $\begin{array}{l}\text { Ratio de penetración en } \\
\text { seguros de no vida }\end{array}$ & NIP & $\begin{array}{l}\text { Primas de seguros de no vida como porcentaje } \\
\text { del PIB. }\end{array}$ & $\begin{array}{l}\text { Swiss Re Swiss Reinsurance } \\
\text { Company (2016) }\end{array}$ \\
\hline & Ratio de penetración total & TIP & $\begin{array}{l}\text { Total de primas de seguros como porcentaje } \\
\text { del PIB. }\end{array}$ & $\begin{array}{l}\text { Swiss Reinsurance Company } \\
(\underline{2016)}\end{array}$ \\
\hline \multirow{5}{*}{$\begin{array}{l}\text { Condiciones } \\
\text { económicas }\end{array}$} & $\begin{array}{l}\text { PIB per cápita, PPC (USD, } \\
\text { precios constantes 2011) }\end{array}$ & GROWTH & $\begin{array}{l}\text { Diferencia logarítmica del PIB per cápita, PPP } \\
\text { (USD, precios constantes 2011). }\end{array}$ & $\begin{array}{l}\text { Calculado a partir de World } \\
\text { Development Indicators } \\
(\underline{2016)}\end{array}$ \\
\hline & Tasa de inflación & INFLA & $\begin{array}{l}\text { Cambio anual en el índice de precios al } \\
\text { consumidor (IPC). }\end{array}$ & $\begin{array}{l}\text { World Development } \\
\text { Indicators }(\underline{2016})\end{array}$ \\
\hline & $\begin{array}{l}\text { Gasto de consumo final de } \\
\text { las administraciones públicas } \\
\text { (\% del PIB) }\end{array}$ & GCONSUMP & $\begin{array}{l}\text { (Log) Ratio de consumo del gobierno sobre el } \\
\text { PIB. }\end{array}$ & $\begin{array}{l}\text { Calculado a partir de World } \\
\text { Development Indicators } \\
(\underline{2016)}\end{array}$ \\
\hline & Comercio (\% del PIB) & TRADE & $\begin{array}{l}\text { Suma de las exportaciones e importaciones de } \\
\text { bienes y servicios medidos como proporción del } \\
\text { PIB. }\end{array}$ & $\begin{array}{l}\text { World Development } \\
\text { Indicators (2016) }\end{array}$ \\
\hline & PIB per cápita inicial & IGROWTH & $\begin{array}{l}\text { (Log) Valor inicial del PIB per cápita, PPC (USD, } \\
\text { precios constantes 2011). }\end{array}$ & $\begin{array}{l}\text { Calculado a partir de World } \\
\text { Development Indicators } \\
(\underline{2016)}\end{array}$ \\
\hline $\begin{array}{l}\text { Condiciones } \\
\text { demográficas }\end{array}$ & $\begin{array}{l}\text { Inscripción escolar, nivel } \\
\text { secundario (\% bruto) }\end{array}$ & HCAPITAL & $\begin{array}{l}\text { Total de la matrícula en educación secundaria } \\
\text { (independientemente de la edad), expresada } \\
\text { como porcentaje de la población en edad de } \\
\text { educación secundaria. }\end{array}$ & $\begin{array}{l}\text { World Development } \\
\text { Indicators }(\underline{2016})\end{array}$ \\
\hline \multirow{3}{*}{ Instituciones } & Estado de derecho & $\mathrm{RL}$ & $\begin{array}{l}\text { Estimación de la gobernabilidad. Oscila entre - } \\
2,5 \text { (débil) y 2,5 (fuerte). }\end{array}$ & $\begin{array}{l}\text { The Worldwide Governance } \\
\text { Indicators (Kaufmann, Kraay y } \\
\text { Mastruzzi } \underline{2010} \text { ) }\end{array}$ \\
\hline & Libertades civiles & $\mathrm{FHCL}$ & $\begin{array}{l}\text { Índice de } 1 \text { a } 7 \text {, donde } 1 \text { representa el mayor } \\
\text { nivel de libertades civiles y } 7 \text { el menor nivel. }\end{array}$ & 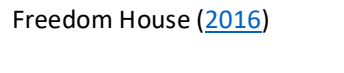 \\
\hline & Derechos políticos & FHPR & $\begin{array}{l}\text { Índice de } 1 \text { a } 7 \text {, donde } 1 \text { representa el mayor } \\
\text { nivel de libertad en derechos políticos y } 7 \text { el } \\
\text { menor nivel. }\end{array}$ & Freedom House (2016) \\
\hline \multirow{3}{*}{$\begin{array}{l}\text { Sistema } \\
\text { financiero }\end{array}$} & $\begin{array}{l}\text { Ratio de la rentabilidad del } \\
\text { mercado de valores }\end{array}$ & TUR & $\begin{array}{l}\text { Ratio del valor total de las acciones negociadas } \\
\text { a la capitalización media real de mercado. El } \\
\text { denominador se deflaciona utilizando el } \\
\text { siguiente método: Tt / P_at / }\left\{(0.5)^{*} \text { [Mt / P_et }\right. \\
\text { + Mt-1 / P_et-1], donde T es el valor total } \\
\text { negociado, M es la capitalización bursátil, P_e } \\
\text { es el IPC de fin de período, P_a es el IPC medio } \\
\text { anual. }\end{array}$ & $\begin{array}{l}\text { Base de datos de desarrollo } \\
\text { financiero, Thorsten Beck. }\end{array}$ \\
\hline & $\begin{array}{l}\text { Valor total del mercado de } \\
\text { valores ( } \% \text { del PIB) }\end{array}$ & STOCKTRA & $\begin{array}{l}\text { Total de acciones cotizadas en el mercado } \\
\text { bursátil respecto al PIB. }\end{array}$ & $\begin{array}{l}\text { Base de datos de desarrollo } \\
\text { financiero, Thorsten Beck. }\end{array}$ \\
\hline & $\begin{array}{l}\text { Crédito privado por depósito } \\
\text { monetario bancario (\% del } \\
\text { PIB) }\end{array}$ & CREDIT & $\begin{array}{l}\text { Crédito privado por bancos de depósitos } \\
\text { bancarios al PIB, calculado utilizando el } \\
\text { siguiente método de deflación: }\{(0,5) *[\mathrm{Ft} / \\
\text { P_et + Ft-1 / P_et-1] }\} \text { / [PIBt / P_at], donde F es } \\
\text { crédito al sector privado, P_e es IPC de fin de } \\
\text { período y P_a es IPC medio anual. }\end{array}$ & $\begin{array}{l}\text { Base de datos de desarrollo } \\
\text { financiero, Thorsten Beck. }\end{array}$ \\
\hline \multirow{2}{*}{ Ingreso } & Ingreso alto & $\mathrm{HI}$ & Países con ingreso alto $=1$, otros $=0$ & Atlas del Banco Mundial \\
\hline & Ingreso medio & MI & Países con ingreso medio $=1$, otros $=0$ & Atlas del Banco Mundial \\
\hline otros & Origen del sistema legal & & $\begin{array}{l}\text { Variable dummy según sea el origen del código } \\
\text { legal (británico, francés, alemán o } \\
\text { escandinavo). }\end{array}$ & $\begin{array}{l}\text { La Porta, Lopez-de-Silanes, } \\
\text { Shleifer y Vishny (1999) }\end{array}$ \\
\hline
\end{tabular}

Fuente: elaboración propia.

Anelena Sabater Castro

(cc) (i) 8 (9)

Revista Economía y Sociedad by Universidad Nacional is licensed under a CreativeCommons Reconocimiento-NoComercial- 
Las variables fundamentales para el estudio son el crecimiento económico, medido por el crecimiento del PIB real per cápita (cuya fuente es el Banco Mundial) y el ratio de penetración de seguros (para vida, no vida y total), extraído de la base de datos facilitada por la Swiss Reinsurance Company. Además, se incluyen como variables de control el gasto de consumo final del gobierno, la tasa de inflación, el ratio de exportaciones e importaciones sobre el PIB y la inscripción escolar en secundaria (esta última como medida de capital humano), cuya fuente es el Banco Mundial.

Cabe señalar que las variables de control se consideran un conjunto de información de las condiciones macroeconómicas de los países. La inclusión de estas variables se fundamenta en los estudios previos, al respecto véase Levine, Loayza y Beck, 2000; Arena, 2008; Chen et al., 2012.

Además, en un segundo modelo o modelo extendido (tal como se especificó en la ecuación (3)) se incluyen variables de las siguientes tres categorías:

1. Ingreso: variables dummy del nivel de ingreso de los países (ingreso alto, medio o bajo), cuya fuente es la clasificación del Banco Mundial. Con la inclusión de estas variables se busca determinar si hay o no diferencia en los resultados al hacer distinción según el nivel de ingreso de los países.

2. Financieras: variables proxies del desarrollo del mercado de valores (ratio de rentabilidad del mercado de valores y valor total del mercado de valores) y una variable proxy del desarrollo del sistema bancario (crédito privado), cuya fuente es la base de datos de desarrollo financiero de Thorsten Beck. La inclusión de estas variables se considera fundamental debido a lo vinculados que están otros componentes del sector financiero y el sector asegurador. Cabe señalar que estas tres variables son frecuentemente utilizadas en la literatura como medidas del desarrollo financiero.

3. Institucionales: variables proxies del desarrollo institucional de los diferentes países (estado de derecho, libertades civiles y derechos políticos). La incorporación de estas variables al modelo permite analizar un área poco explorada por la literatura científica. Se consideró como referencia para la selección de las variables el estudio de Lee, Chang, Aurouri y Lee (2016) y los datos disponibles en The Worldwide Governance Indicators de Kaufmann et al. (2010) y Freedom House (2016).

En ese segundo modelo se incluyen además los términos de interacción entre las variables anteriores y el ratio de penetración de seguros (vida, no vida y total), tal y como se especificó en la ecuación (3). El propósito es analizar si -y qué medida- estas variables condicionan (refuerzan o mitigan) la relación entre el desarrollo del sector asegurador y el crecimiento económico.

Por otro lado, cabe señalar que los instrumentos considerados para las estimaciones son las mismas variables rezagadas tanto en diferencias como en niveles. Asimismo, siguiendo a Arena (2008), se incluye el origen del código legal (británico, alemán, francés o escandinavo) como 96

Anelena Sabater Castro

(c) (1) 8 (2)

Revista Economía y Sociedad by Universidad Nacional is licensed under a CreativeCommons Reconocimiento-NoComercial- 
instrumento de las variables financieras. El origen del código legal se considera exógeno y, por ello, funge como variable instrumental que permite controlar el sesgo de simultaneidad, tal como señalan Levine et al. (2000).

\section{Resultados}

\subsection{Modelo básico}

En la Tabla $\underline{2}$ se muestran los resultados de las estimaciones del modelo básico, que corresponden con la ecuación (2). Inicialmente, cabe destacar que las tres estimaciones presentan el coeficiente de la variable PIB per cápita inicial estadísticamente significativo, lo cual implica que el panel es dinámico. Además, en los test de correlación serial y el test de Sargan no se rechaza la hipótesis nula de especificación correcta, lo cual respalda los resultados obtenidos.

La columna 1 de la Tabla $\underline{2}$ muestra el efecto del ratio de penetración de seguros de vida (LIP) en el crecimiento económico. Dado que el coeficiente de LIP es positivo y estadísticamente significativo esto sugiere que el desarrollo del mercado asegurador del segmento de vida tiene un efecto positivo en el crecimiento económico (medido por la variable proxy LIP). Por su parte, en la columna 2 se muestra el efecto del ratio de penetración de seguros de no vida (NIP) en el crecimiento económico, cuyo coeficiente también es positivo y estadísticamente significativo, lo cual sugiere que el desarrollo del mercado asegurador del segmento de no vida tiene un efecto positivo en el crecimiento económico. Por último, en la columna 3 se muestra el efecto del ratio de penetración de seguros total (TIP) en el crecimiento económico, coeficiente que también resulta positivo y estadísticamente significativo.

Respecto al efecto individual de cada variable, el incremento en una desviación estándar en el ratio de seguros de vida, ceteris paribus, implica el aumento de $0,37 \%$ en el crecimiento de una economía ${ }^{10}$. Por su parte, en cuanto al ratio de seguros de no vida, el incremento de una desviación estándar en este, ceteris paribus, tiene un impacto de 0,20\% en el crecimiento de una economía. Finalmente, el incremento de una desviación estándar en el ratio total de seguros aumenta el crecimiento del PIB per cápita real en 0,42\%.

Los resultados sustentan las conclusiones de estudios previos, Arena (2008), Haiss and Sumegi (2008) y Chen et al. (2012), en el sentido de que la actividad aseguradora en el segmento de vida tiene un impacto positivo en el crecimiento económico. Respecto al segmento de no vida los resultados son similares a los hallazgos de Arena (2008).

\footnotetext{
${ }^{10}$ Se refiere a los coeficientes de regresión estandarizados, los cuales permiten mayor comparabilidad. En este caso se han realizado las estimaciones expresando todas las variables de la regresión en forma tipificada (puntuaciones z).
} 
Tabla 2.

Resultados: estimación básica

\begin{tabular}{|c|c|c|c|}
\hline Variables explicativas & 1 & 2 & 3 \\
\hline \multirow[t]{2}{*}{ IGROWTH } & $-0,0164 * * *$ & $-0,0173 * * *$ & $-0,0190 * * *$ \\
\hline & 0,0000 & 0,0000 & 0,0000 \\
\hline \multirow[t]{2}{*}{ LIP } & $0,3410 * * *$ & & \\
\hline & 0,0000 & & \\
\hline \multirow[t]{2}{*}{ NIP } & & $0,3432 *$ & \\
\hline & & 0,0690 & \\
\hline \multirow[t]{2}{*}{ TIP } & & & $0,3146 * * *$ \\
\hline & & & 0,0000 \\
\hline \multicolumn{4}{|l|}{ Variables de control } \\
\hline \multirow[t]{2}{*}{ INFLA } & 0,0002 & 0,0002 & $0,0003^{*}$ \\
\hline & 0,1760 & 0,1010 & 0,0700 \\
\hline \multirow[t]{2}{*}{ TRADE } & $0,0107 * * *$ & $0,0112 * * *$ & $0,0079 * *$ \\
\hline & 0,0070 & 0,0000 & 0,0440 \\
\hline \multirow[t]{2}{*}{ GCONSUMP } & $-0,1139 * * *$ & $-0,0952 * * *$ & $-0,1229 * * *$ \\
\hline & 0,0000 & 0,0020 & 0,0000 \\
\hline \multirow[t]{2}{*}{ HCAPITAL } & $0,0006 * * *$ & $0,0006 * * *$ & $0,0007^{* * *}$ \\
\hline & 0,0000 & 0,0000 & 0,0000 \\
\hline \multicolumn{4}{|l|}{ Períodos } \\
\hline \multirow[t]{2}{*}{ 1995-1999 } & 0,0012 & $-0,0001$ & 0,0002 \\
\hline & 0,6940 & 0,9660 & 0,9470 \\
\hline \multirow[t]{2}{*}{ 2000-2004 } & $0,0061 * * *$ & $0,0050 * * *$ & $0,0054 * * *$ \\
\hline & 0,0020 & 0,0000 & 0,0000 \\
\hline \multirow[t]{2}{*}{ 2005-2009 } & $-0,0051 * * *$ & $-0,0056 * * *$ & $-0,0052 * * *$ \\
\hline & 0,0000 & 0,0000 & 0,0000 \\
\hline \multirow[t]{2}{*}{$2010-2014$} & $-0,0091 * * *$ & $-0,0117 * * *$ & $-0,0095 * * *$ \\
\hline & 0,0000 & 0,0000 & 0,0000 \\
\hline Países & 47 & 47 & 47 \\
\hline \multirow{2}{*}{\multicolumn{4}{|c|}{$\begin{array}{l}\text { Observaciones } \\
\text { Pruebas de especificación }\end{array}$}} \\
\hline & & & \\
\hline $\mathrm{AR}(1)$ & 0,0190 & 0,0130 & 0,0190 \\
\hline $\operatorname{AR}(2)$ & 0,4450 & 0,3830 & 0,4820 \\
\hline Test de Hansen & 0,1800 & 0,1270 & 0,3630 \\
\hline
\end{tabular}

Notas: Consultar Tabla 1 para ver las abreviaturas de las variables. $A R(1)$ y $A R(2)$ denota el test Arellano-Bond de primer y segundo orden en primeras diferencias. Variable dependiente: crecimiento real del PIB per cápita. P-values en cursiva. $* * *, * * y *$ denotan significatividad al $1 \%, 5 \%$ y $10 \%$.

Fuente: elaboración propia.

\subsection{Modelo extendido: variables de ingreso}

En la Tabla $\underline{3}$ se muestran los resultados utilizando variables dicotómicas de tres niveles de ingresos: alto, medio y bajo. Tal como se observa, los coeficientes para los países altos (columna 1) son todos estadísticamente significativos $y$, al ser el coeficiente del término de interacción (TIP*HI) negativo, esto sugiere que los países con ingresos altos están mitigando el efecto 98

Anelena Sabater Castro

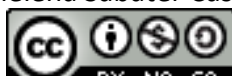

Revista Economía y Sociedad by Universidad Nacional is licensed under a CreativeCommons Reconocimiento-NoComercial- 
positivo del desarrollo del mercado asegurador, respecto a los países de ingreso bajo. Es decir, mientras que un mayor desarrollo del mercado asegurador promueve el crecimiento económico, este efecto positivo se ve aminorado en los países de ingresos altos.

Estos resultados son hasta cierto punto esperables y coherentes con la teoría económica, ya que en los países más desarrollados, cuyos niveles de ingreso son altos, el mercado asegurador está, en general, bastante desarrollado, por tanto la contribución de la actividad aseguradora al crecimiento económico ha alcanzado ya niveles altos. Este hallazgo es señalado por otros autores (Arena (2008) y Herce et al. (2013)). En cuanto a los países con ingresos medios (columna 2) la no significancia del coeficiente de interacción impide realizar interpretaciones, indicando que para este conjunto de países el efecto no presenta significancia. Esto puede indicar -concretamente con los datos del presente estudio- que la variable de ingreso para el caso de los países de ingreso medio no muestra resultados robustos.

Tabla 3.

Resultados: variables de ingreso

\begin{tabular}{lcc}
\hline Variables explicativas & $\mathbf{1}$ & $\mathbf{2}$ \\
\hline TIP & $0,2217^{* * *}$ & 0,0435 \\
& 0,0010 & 0,6680 \\
TIP*HI & $-0,2865^{* *}$ & \\
& 0,0270 & \\
HI & $0,0606^{* *}$ & $0,0438^{*}$ \\
& 0,0140 & 0,0560 \\
TIP*MI & & 0,1317 \\
& & 0,2820 \\
MI & $0,0171^{*}$ & 0,0146 \\
& 0,0880 & 0,1420 \\
Pruebas de especificación & & \\
AR(1) & 0,0220 & 0,0220 \\
AR(2) & 0,7690 & 0,7190 \\
Test de Hansen & 0,1310 & 0,1260 \\
\hline
\end{tabular}

Notas: Variable dependiente: crecimiento real del PIB per cápita. P-values en cursiva. $* * *, * * \mathrm{y} *$ denotan significatividad al $1 \%, 5 \%$ y $10 \%$.

Fuente: elaboración propia.

\subsection{Modelo extendido: variables institucionales}

En la Tabla $\underline{4}$ se muestran los resultados de las estimaciones al incluir en el modelo las variables institucionales y sus correspondientes interacciones con las variables proxies del desarrollo del mercado asegurador.

Las variables incluidas son estado de derecho ( $R L$ ), en las columnas 1-3; derechos políticos (FHPR), en las columnas 4-6; y libertades civiles (FHCL), en las columnas 7-9. En relación con los resultados, se debe señalar que aquellos coeficientes de las variables proxies del entorno 
institucional que resultaron estadísticamente significativos tienen signo positivo. Ello sugiere que el desarrollo institucional afecta positivamente el crecimiento económico.

Por otro lado, al observar los resultados se puede apreciar que estos son sustancialmente diferentes según el segmento al que se refieran. Respecto al segmento de no vida, se aprecia que el término de interacción entre la variable ratio de penetración de seguros y la variable institucional en todos los casos resulta estadísticamente significativo y negativo. Esto sugiere que las variables institucionales incluidas están afectando negativamente el efecto positivo del mercado asegurador -segmento de no vida- en el crecimiento económico.

Estos resultados sugieren que, cuando una economía tiene instituciones sólidas o un nivel institucional desarrollado -en términos de las variables utilizadas-, el efecto del desarrollo del mercado asegurador (concretamente de no vida) sobre el crecimiento económico se ve mitigado. A resultados similares llegan Lee et al. (2016) para el segmento de vida, al indicar que cuando los entornos institucionales son sólidos para que todos los sectores dentro de la economía puedan funcionar bien, la contribución del sector de seguros puede no ser significativa.

En el caso del segmento de vida, el término de interacción entre la ratio de penetración de seguros y la variable institucional no resulta estadísticamente significativo en la mayoría de las estimaciones. Por ello se considera que los resultados no permiten generar interpretaciones robustas acerca de este segmento.

Los resultados permiten poner de manifiesto que existe una necesidad latente de estudios que busquen determinar empíricamente el rol de las instituciones en el vínculo desarrollo del sector asegurador y crecimiento económico.

\subsection{Modelo extendido: variables financieras}

En Apéndice $\underline{2}$ se muestran los resultados de analizar las variables financieras y sus correspondientes interacciones con las variables de seguros. Tal como se indicó en el apartado 2.2, las variables incluidas son: el valor total del mercado de valores (STOCKTRA) y el ratio de rentabilidad del mercado de valores (TUR)) y el crédito privado por depósito monetario bancario (CREDIT).

En el caso de STOCKTRA, los resultados se observan en las columnas 1-3. En las tres estimaciones realizadas el coeficiente de interacción entre el ratio de penetración de seguros y STOCKTRA es significativo y negativo, lo cual indica que el incremento en el ratio de penetración de seguros (según sea de vida, no vida o total) aumenta el crecimiento económico; sin embargo, el efecto positivo se ve afectado negativamente por STOCKTRA.

100

Anelena Sabater Castro

(c) (i) 8 (2)

Revista Economía y Sociedad by Universidad Nacional is licensed under a CreativeCommons Reconocimiento-NoComercial- 
Respecto a TUR, los resultados se muestran en las columnas 4-6. En el caso de vida y no vida (columnas 4 y 5) el coeficiente de la interacción no se puede interpretar, al no ser estadísticamente significativo. Sin embargo, en el caso del término de interacción entre el ratio de penetración de seguro total y TUR este sí es estadísticamente significativo y negativo, lo cual sugiere que a nivel total (es decir, ambos segmentos de seguros) el desarrollo de mercado de seguros tiene un impacto positivo en el crecimiento económico y el término TUR afecta este impacto negativamente.

Por último, los resultados de las estimaciones al incluir la variable CREDIT se muestran en las columnas 7-9. En las tres estimaciones el coeficiente de interacción entre el ratio de penetración de seguros y CREDIT es significativo y negativo. Similar al análisis de las variables STOCKTRA y TUR, los resultados sugieren que el desarrollo de mercado de seguros tiene un impacto positivo en el crecimiento económico; no obstante, el crédito privado está afectando este impacto de forma adversa. En términos generales, los resultados anteriormente expuestos implican un posible efecto sustitución, entre el desarrollo del mercado asegurador y el financiero.

\section{Tabla 4.}

Resultados: variables institucionales

\begin{tabular}{|c|c|c|c|c|c|c|c|c|c|}
\hline $\begin{array}{l}\text { Variables } \\
\text { explicativas }\end{array}$ & 1 & 2 & 3 & 4 & 5 & 6 & 7 & 8 & 9 \\
\hline \multirow[t]{2}{*}{ IGROWTH } & $-0,0349 * * *$ & $-0,0331 * * *$ & $-0,0333^{* * *}$ & $-0,0207^{* * *}$ & $-0,0220 * * *$ & $-0,0204 * * *$ & $-0,0231^{* * *}$ & $-0,0231^{* * *}$ & $-0,0209 * * *$ \\
\hline & 0,0000 & 0,0000 & 0,0000 & 0,0000 & 0,0000 & 0,0000 & 0,0000 & 0,0000 & 0,0000 \\
\hline \multirow[t]{2}{*}{ LIP } & 0,0369 & & & 0,0824 & & & $-0,0564$ & & \\
\hline & 0,7410 & & & 0,1190 & & & 0,7720 & & \\
\hline \multirow[t]{2}{*}{ NIP } & & $0,8607 * * *$ & & & $0,8248^{* * *}$ & & & $0,9004^{* * *}$ & \\
\hline & & 0,0020 & & & 0,0070 & & & 0,0050 & \\
\hline \multirow[t]{2}{*}{ TIP } & & & $0,2221 * * *$ & & & 0,2830 & & & $0,3283^{* * *}$ \\
\hline & & & 0,0070 & & & 0,9620 & & & 0,0000 \\
\hline \multirow[t]{2}{*}{ LIP*RL } & 0,7410 & & & & & & & & \\
\hline & 0,8960 & & & & & & & & \\
\hline \multirow[t]{2}{*}{$N I P * R L$} & & $-0,4399 * * *$ & & & & & & & \\
\hline & & 0,001 & & & & & & & \\
\hline \multirow[t]{2}{*}{ TIP*RL } & & & $-0,1490 * * *$ & & & & & & \\
\hline & & & 0,0080 & & & & & & \\
\hline \multirow[t]{2}{*}{$\mathrm{RL}$} & $0,0183^{* * *}$ & $0,0303^{* * *}$ & $0,0248 * * *$ & & & & & & \\
\hline & 0,0000 & 0,0000 & 0,0000 & & & & & & \\
\hline \multirow[t]{2}{*}{ LIP*FHPR } & & & & $0,1086 * *$ & & & & & \\
\hline & & & & 0,0110 & & & & & \\
\hline \multirow[t]{2}{*}{ NIP*FHPR } & & & & & $-0,3977^{* *}$ & & & & \\
\hline & & & & & 0,0130 & & & & \\
\hline \multirow[t]{2}{*}{ TIP*FHPR } & & & & & & $-0,0019^{* *}$ & & & \\
\hline & & & & & & 0,0200 & & & \\
\hline \multirow[t]{2}{*}{ FHPR } & & & & $-0,0004$ & $0,0057^{* *}$ & $0,0024^{*}$ & & & \\
\hline & & & & 0,7810 & 0,0170 & 0,0980 & & & \\
\hline \multirow[t]{2}{*}{ LIP*FHCL } & & & & & & & 0,1531 & & \\
\hline & & & & & & & 0,1860 & & \\
\hline NIP*FHCL & & & & & & & & $-0,4527 * * *$ & \\
\hline
\end{tabular}




\begin{tabular}{|c|c|c|c|c|c|c|c|c|c|}
\hline $\begin{array}{l}\text { Variables } \\
\text { explicativas }\end{array}$ & 1 & 2 & 3 & 4 & 5 & 6 & 7 & 8 & 9 \\
\hline & & & & & & & & 0,0020 & \\
\hline \multirow[t]{2}{*}{ TIP*FHCL } & & & & & & & & & $-0,0377$ \\
\hline & & & & & & & & & 0,3830 \\
\hline \multirow[t]{2}{*}{$\mathrm{FHCL}$} & & & & & & & $-0,0024$ & $0,0062 * * *$ & 0,0032 \\
\hline & & & & & & & 0,1790 & 0,0090 & 0,1470 \\
\hline \multicolumn{10}{|c|}{ Variables de control } \\
\hline \multirow[t]{2}{*}{ INFLA } & $0,0004 * *$ & $0,0005^{* * *}$ & $0,0004 * *$ & 0,0002 & 0,0002 & $0,0003 * *$ & 0,0002 & 0,0001 & 0,0002 \\
\hline & 0,0320 & 0,0020 & 0,0450 & 0,1370 & 0,2560 & 0,0310 & 0,1800 & 0,5450 & 0,1730 \\
\hline \multirow[t]{2}{*}{ TRADE } & 0,0041 & $-0,0033$ & 0,0013 & $0,0181^{* * *}$ & $0,0097^{* * *}$ & $0,0137^{* * *}$ & $0,0194^{* * *}$ & $0,0114 * * *$ & $0,0123 * * *$ \\
\hline & 0,3040 & 0,2810 & 0,6810 & 0,0000 & 0,0000 & 0,0000 & 0,0000 & 0,0000 & 0,0000 \\
\hline \multirow[t]{3}{*}{ GCONSUMP } & - & - & - & - & - & - & $-0,0500 * *$ & - & - \\
\hline & $0,1161^{* * *}$ & $0,1676 * * *$ & $0,1341 * * *$ & $0,0718 * * *$ & $0,0844 * * *$ & $0,1070 * * *$ & & $0,0966 * * *$ & $0,1097 * * *$ \\
\hline & 0,0000 & 0,0000 & 0,0000 & 0,0000 & 0,0000 & 0,0000 & 0,0180 & 0,0000 & 0,0000 \\
\hline \multirow[t]{2}{*}{ HCAPITAL } & $0,0008 * * *$ & $0,0007^{* * *}$ & $0,0007 * * *$ & $0,0006 * * *$ & $0,0008 * * *$ & $0,0007 * * *$ & $0,0005^{* * *}$ & $0,0008 * * *$ & $0,0007^{* * *}$ \\
\hline & 0,0000 & 0,0000 & 0,0000 & 0,0000 & 0,0000 & 0,0000 & 0,0000 & 0,0000 & 0,0000 \\
\hline \multicolumn{10}{|l|}{ Períodos } \\
\hline \multirow[t]{3}{*}{ 1995-1999 } & $-0,0053^{*}$ & - & $-0,0065^{* *}$ & 0,0037 & 0,0029 & $0,0083 * * *$ & 0,0035 & $0,0099 * * *$ & 0,0032 \\
\hline & & $0,0099 * * *$ & & & & & & & \\
\hline & 0,0690 & 0,0000 & 0,0460 & 0,1660 & 0,1680 & 0,0010 & 0,3230 & 0,0000 & 0,2060 \\
\hline \multirow[t]{2}{*}{ 2000-2004 } & $0,0032 *$ & 0,0011 & 0,0029 & $0,0079 * * *$ & $0,0056 * * *$ & $0,0127 * * *$ & $0,0075^{* * *}$ & $0,0112 * * *$ & $0,0063 * * *$ \\
\hline & 0,0880 & 0,5110 & 0,1590 & 0,0000 & 0,0000 & 0,0000 & 0,0010 & 0,0000 & 0,0040 \\
\hline \multirow[t]{3}{*}{ 2005-2009 } & - & - & - & - & - & $0,0060 * * *$ & - & $0,0052 * * *$ & - \\
\hline & $0,0035^{* * *}$ & $0,0036 * * *$ & $0,0032 * * *$ & $0,0052 * * *$ & $0,0047^{* * *}$ & & $0,0048 * * *$ & & $0,0049 * * *$ \\
\hline & 0,0080 & 0,0020 & 0,0080 & 0,0000 & 0,0010 & 0,0000 & 0,0000 & 0,0010 & 0,0010 \\
\hline \multirow[t]{3}{*}{$2010-2014$} & $-0,0094^{*}$ & - & - & - & - & - & - & - & - \\
\hline & & $0,0088 * * *$ & $0,0089 * * *$ & $0,0108 * * *$ & $0,0099 * * *$ & $0,0048 * * *$ & $0,0098 * * *$ & $0,0062 * * *$ & $0,0104 * * *$ \\
\hline & 0,0700 & 0,0000 & 0,0000 & 0,0000 & 0,0000 & 0,0000 & 0,0700 & 0,0000 & 0,0000 \\
\hline Países & 47 & 47 & 47 & 47 & 47 & 47 & 47 & 47 & 47 \\
\hline Observacione & 231 & 231 & 231 & 231 & 231 & 231 & 231 & 231 & 231 \\
\hline $\mathrm{s}$ & & & & & & & & & \\
\hline \multicolumn{10}{|c|}{ Pruebas de especificación } \\
\hline $\operatorname{AR}(1)$ & 0,0160 & 0,0220 & 0,0160 & 0,0170 & 0,0090 & 0,0240 & 0,0140 & 0,0090 & 0,0170 \\
\hline$A R(2)$ & 0,7260 & 0,8900 & 0,7470 & 0,3340 & 0,2200 & 0,5550 & 0,4100 & 0,1270 & 0,3210 \\
\hline $\begin{array}{l}\text { Test de } \\
\text { Hansen }\end{array}$ & 0,2090 & 0,1960 & 0,1980 & 0,2040 & 0,2460 & 0,1930 & 0,2520 & 0,2980 & 0,1950 \\
\hline
\end{tabular}

Notas: En la Tabla 1 se presentan las abreviaturas de las variables. AR(1) y AR(2) denota el test ArellanoBond de primer y segundo orden en primeras diferencias. Las estimaciones también incluyen las correspondientes variables dicotómicas de los cinco periodos de tiempo. Variable dependiente: crecimiento real del PIB per cápita. P-values en cursiva. ${ }^{* * *}, * *$ y $*$ denotan significatividad al $1 \%, 5 \%$ y $10 \%$.

Fuente: elaboración propia.

\section{Discusión}

El mercado asegurador cumple dentro de una economía diversas funciones que resultan de suma relevancia, por lo cual se habla de que su importancia es estratégica en el ámbito social y económico. No obstante, a diferencia de otros sectores, el vínculo entre el mercado asegurador y el crecimiento económico a nivel empírico no ha sido vastamente explotado. Ante ello este estudio aporta resultados empíricos tanto para el segmento de vida como para no vida e incluye en el estudio variables institucionales.

102

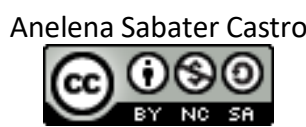

Revista Economía y Sociedad by Universidad Nacional is licensed under a CreativeCommons Reconocimiento-NoComercial- 
A nivel general, los resultados sugieren que el desarrollo del mercado asegurador (tanto de vida como de no vida) tiene un efecto positivo sobre el crecimiento económico. Es decir, es posible afirmar que el aumento en el volumen de primas de seguros de vida y no vida tiene un efecto positivo y significativo en el crecimiento económico. Esto es trascendental, desde un punto de vista microeconómico, un mercado asegurador solido e incluyente permite administrar los riesgos de manera eficiente y, por ende, fomenta la productividad de las empresas y hogares. De manera tal que estos agentes económicos busquen oportunidades con mayor riesgo y mayor retorno. Además, los seguros fungen como herramienta de política pública, ya que promueven el acceso a servicios básicos (como la salud) y reducen la vulnerabilidad (por ejemplo, a través del microseguro). Este impacto positivo de los seguros en los agentes económicos se traduce entonces en crecimiento económico.

Por otro lado, los resultados son aún más relevantes para países de ingresos bajos, en los cuales la contribución de más actividad aseguradora al crecimiento económico es mayor en comparación con los países de niveles de ingreso alto. Esto se vincula con que los países de ingresos altos tienen, en general, un mercado asegurador bastante profundo; por tanto, la contribución de la actividad aseguradora al crecimiento económico ha alcanzado ya niveles importantes.

Por su parte, al analizar la interacción de diversas variables financieras con el desarrollo del mercado asegurador es posible concluir que, en general, hay un efecto de interacción negativo y estadísticamente significativo. Lo que representa un posible efecto sustitutivo entre el desarrollo del sector bancario y el sector asegurador, tema que ha sido abordado por otros autores, cabe destacar a Chen et al. (2012). Además, se debe poner de manifiesto la necesidad de investigaciones que busquen determinar empíricamente si el desarrollo del mercado asegurador y el desarrollo financiero son sustitutos y, de ser así, a partir de qué nivel de desarrollo financiero.

Los resultados de las estimaciones al incluir las variables institucionales permiten concluir que, cuando una economía tiene instituciones sólidas o un nivel institucional desarrollado, esto aminora el efecto positivo del desarrollo del mercado asegurador (específicamente para los seguros de no vida) sobre el crecimiento económico. Más concretamente, cuando los países tienen instituciones sólidas el efecto de un mayor desarrollo asegurador sobre el crecimiento económico se ve mitigado, lo cual es congruente con la intuición, pues las economías desarrolladas se esperaría que tengan un entramado institucional fuerte.

A la luz de los resultados y de la discusión anteriormente planteada es fundamental agregar que los gobiernos deben realizar acciones estratégicas con el fin de incentivar el acceso a los seguros; es decir, incrementar la penetración de los seguros, fomentando 1) un mercado de seguros inclusivo (desde una perspectiva de demanda) y 2) la cultura de previsión de riesgos. Esto a la vez contribuye a mejorar la cultura financiera de las personas. En términos de políticas 
públicas, resulta, por tanto, de suma relevancia fomentar el ahorro por medio del seguro. Tal como se señaló en secciones previas, las compañías aseguradoras son importantes inversionistas institucionales y por ejemplo pueden facilitar la inversión pública por medio del financiamiento de deuda emitida por el Estado.

Además, cabe señalar que actualmente se están gestando cambios importantes en materia regulatoria para las compañías aseguradoras y es trascendental su implementación, pues permitirá fortalecer la capacidad financiera de las instituciones y desarrollar mercados más sanos.

Por último, se ponen de manifiesto algunas recomendaciones para estudios posteriores: 1) la inclusión de otras variables proxies del desarrollo del mercado asegurador -por ejemplo la densidad del aseguramiento- , 2) es crucial considerar el tema de la heterogeneidad de los países al analizar el sector asegurador y el crecimiento económico y 3) incorporar variables que puedan captar los efectos de la regulación en el ámbito asegurador y el rol de estas en el vínculo entre el desarrollo asegurador y el crecimiento económico.

\section{Referencias}

Arellano, M. \& Bond, S. (1991). Some Tests of Specification for Panel Data: Monte Carlo Evidence and an Application to Employment Equations. The Review of Economic Studies, 58(2), 277-297. Recuperado de https://doi.org/10.2307/2297968

Arellano, M. \& Bover, O. (1995). Another Look at the Instrumental-Variable Estimation of ErrorComponents Models. Journal of Econometrics, 68(1), 29-51. Recuperado de https://doi.org/10.1016/0304-4076(94)01642-D

Arena, M. (2008). Does Insurance Market Activity Promote Economic Growth? A Cross-Country Study For Industrialized and Developing Countries. The Journal of Risk and Insurance, 75(4), 921-946. Recuperado de http://dx.doi.org/10.1111/j.1539-6975.2008.00291.x

Barr, N. (2010). Long-term care: a suitable case for social insurance. Social Policy \& Administration, 44(4), 359-374. Recuperado de http://dx.doi.org/10.1111/j.14679515.2010.00718.x

Blundella, R. \& Bond, S. (1998). Initial Conditions and Moment Restrictions in Dynamic Panel Data Models. Journal of Econometrics, 87(1), 115-143. Recuperado de https://doi.org/10.1016/S0304-4076(98)00009-8

104

Anelena Sabater Castro

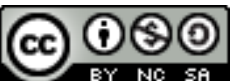

Revista Economía y Sociedad by Universidad Nacional is licensed under a CreativeCommons Reconocimiento-NoComercial- 
Chamberlain, D. Coetze, W., \& Camargo, A. (2017). Funding the Frontier: The Link Between Inclusive Insurance Market, Growth and Poverty Reduction in Africa. Recuperado de http://cenfri.org/microinsurance/assuring-growth-the-link-between-inclusive-insurancemarkets-growth-and-poverty-reduction

Chen, P-F., Lee, C-C., \& Lee, C-F. (2012). How does the development of the life insurance market affect economic growth? Some international evidence. Journal International Development, 24(7), 865-893. Doi: https://doi.org/10.1002/jid.1765

Dercon, S. \& Christiaensen, L. (2007). Consumption Risk, Technology Adoption and Poverty Traps: Evidence from Ethiopia (Working paper No. 4257). Recuperado de http://documentos.bancomundial.org/curated/es/703071468256451288/Consumptionrisk-technology-adoption-and-poverty-traps-evidence-from-Ethiopia

Feyen, E., Lester, R., \& Rocha, R. (2011). What Drives the Development of the Insurance Sector? An Empirical Analysis Based on a Panel of Developed and Developing Countries. Policy Research (Working Paper No. 5572). Recuperado de https://openknowledge.worldbank.org/handle/10986/3339

Focarelli, D. (2017). Why insurance regulation is crucial for long-term investment and economic growth (School of European Political Economy, Working Paper No.1). Recuperado de http://sep.luiss.it/research/working-papers/2017/01/13/d-focarelli-why-insuranceregulation-crucial-long-term-investment

Freedom House (2016). Database, Freedom in the World. Washington, DC. Recuperado de https://freedomhouse.org/report/freedom-world-2016/table-scores

Haiss, P. \& Sumegi, K. (2008). The Relationship of Insurance and Economic Growth: a Theoretical and Empirical Analysis. Empirica 35(4), 405-431. Doi: https://doi.org/10.1007/s10663$\underline{008-9075-2}$

Herce, J., Delgado, D., Azpeitia, F., Blasco, I., \& Hernández, P. (2013). El seguro en la sociedad y economía españolas, balance socieconómico de una industria necesaria. Fundación Mapfre. Madrid, España. Recuperado de https://www.fundacionmapfre.org/documentacion/publico/es/catalogo imagenes/grup o.cmd?path $=1075909$

Insurance Europe. (2016). European insurance industry database. Bruselas, Bélgica. Recuperado de https://insuranceeurope.eu/european-insurance-industry-database 
Kaufmann, D., Kraay, A., \& Mastruzzi, M. (2010). The Worldwide Governance Indicators. World Bank: Washington, DC. Recuperado de http://info.worldbank.org/governance/wgi/\#home

La Porta, R., Lopez-de-Silanes, F., Shleifer, A., \& Vishny, R. (1999). The Quality of Government. Journal of Law, Economics, and Organization, 15(1), 222-279. Doi: http://dx.doi.org/10.2139/ssrn.183908

Lee, C-C., Chang, C-H., Arouri, M., \& Lee, C-C. (2016). Economic growth and insurance development: The role of institutional environments. Economic Modelling, 59, 361-369. Doi: https://doi.org/10.1016/j.econmod.2016.08.010

Levine, R., Loayza, N., \& Beck, T. (2000). Financial Intermediation and Growth: Causality and Causes. Journal of Monetary Economics, 46(1), 31-77. Recuperado de https://doi.org/10.1016/S0304-3932(00)00017-9

Organization for Economic Cooperation and Development. (2016). Insurance companies and employees. Doi: http://dx.doi.org/10.1787/21c971c6-en

Outreville, F. (1998). Theory and Practice of Insurance. Boston: Kluwer Academic Publishers. Doi: http://dx.doi.org/10.1007/978-1-4615-6187-3

Outreville, F. (2013). The Relationship between Insurance and Economic Development: 85 Empirical Papers for a Review of the Literature. Risk Management and Insurance Review, 16(1), 71-122. Doi: http://dx.doi.org/\%2010.1111/j.1540-6296.2012.01219.x

Roodman, D. (2009). How to Do xtabond2: An Introduction to "Difference" and "System" GMM in Stata. Stata Journal 9(1), 86-136. Recuperado de https://econpapers.repec.org/article/tsjstataj/v 3a9 3ay 3a2009 3ai 3a1 3ap 3a86136.htm

Swiss Reinsurance Company. (2016) World Insurance in 2015: Growing Premiums and Stronger Balance Sheets. Zúrich, Suiza: Suministrado por Swiss Re.

World Development Indicators. (2016). World Development Indicators Featuring the Sustainable Development Goals. Recuperado de http://databank.worldbank.org/data/download/site-content/wdi-2016-highlightsfeaturing-sdgs-booklet.pdf

106

Anelena Sabater Castro

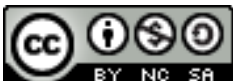

Revista Economía y Sociedad by Universidad Nacional is licensed under a CreativeCommons Reconocimiento-NoComercial- 
Economía y Sociedad, Vol. 22, № 52, julio-diciembre 2017, pp. 88-109

\section{Apéndice}

Apéndice 1.

Países incluidos en el análisis

\begin{tabular}{|c|c|c|}
\hline Ingreso alto (23) & Ingreso medio (13) & Ingreso bajo (11) \\
\hline Alemania & Argentina & Bangladesh \\
\hline Australia & Brasil & Egipto \\
\hline Austria & China & El Salvador \\
\hline Bélgica & Colombia & Filipinas \\
\hline Canadá & Ecuador & India \\
\hline Chile & Irán & Indonesia \\
\hline Chipre & Malasia & Kenia \\
\hline Dinamarca & México & Marruecos \\
\hline España & Panamá & Nigeria \\
\hline Estados Unidos & Sudáfrica & Pakistán \\
\hline Finlandia & Tailandia & Túnez \\
\hline Francia & Turquía & \\
\hline Grecia & Venezuela & \\
\hline \multicolumn{3}{|l|}{ Irlanda } \\
\hline \multicolumn{3}{|l|}{ Islandia } \\
\hline \multicolumn{3}{|l|}{ Israel } \\
\hline \multicolumn{3}{|l|}{ Italia } \\
\hline \multicolumn{3}{|l|}{ Japón } \\
\hline \multicolumn{3}{|l|}{ Países Bajos } \\
\hline \multicolumn{3}{|l|}{ Portugal } \\
\hline \multicolumn{3}{|l|}{ Reino Unido } \\
\hline \multicolumn{3}{|l|}{ Suecia } \\
\hline \multicolumn{3}{|l|}{ Suiza } \\
\hline $\begin{array}{l}\text { Nota: Clasificació } \\
\text { clasificados en } \\
\text { US\$4,035 en el In } \\
\text { Fuente: Elaboraci }\end{array}$ & $\begin{array}{l}\text { Banco Mundial a } \mathrm{m} \\
\text { so bajo son aque }\end{array}$ & $\begin{array}{l}\text { arzo 2017. Los paíse } \\
\text { los con menos c } \\
\text { cápita. }\end{array}$ \\
\hline
\end{tabular}


Economía y Sociedad, Vol. 22, № 52, julio-diciembre 2017, pp. 88-109

Apéndice 2.

Resultados: variables financieras

\begin{tabular}{|c|c|c|c|c|c|c|c|c|c|}
\hline $\begin{array}{l}\text { Variables } \\
\text { explicativas }\end{array}$ & 1 & 2 & 3 & 4 & 5 & 6 & 7 & 8 & 9 \\
\hline \multirow[t]{2}{*}{ IGROWTH } & $-0,0244^{* * *}$ & $-0,0193 * * *$ & $-0,0205^{* * *}$ & $-0,0208 * * *$ & $-0,0241 * * *$ & $-0,0197 * * *$ & $-0,0145 * * *$ & $-0,0153^{* * *}$ & $-0,0157^{* * *}$ \\
\hline & 0,0000 & 0,0000 & 0,0000 & 0,0000 & 0,1240 & 0,0000 & 0,0000 & 0,0000 & 0,0000 \\
\hline \multirow[t]{2}{*}{ LIP } & $0,2750^{* * *}$ & & & $0,2905^{* * *}$ & & & $0,4622^{* * *}$ & & \\
\hline & 0,0000 & & & 0,0010 & & & 0,0000 & & \\
\hline \multirow[t]{2}{*}{ NIP } & & $1,1895^{* * *}$ & & & $0,5230 * * *$ & & & $1,4383^{* * *}$ & \\
\hline & & 0,0000 & & & 0,0010 & & & 0,0000 & \\
\hline \multirow[t]{2}{*}{ TIP } & & & $0,3206 * * *$ & & & $0,3211^{* * *}$ & & & $0,3973 * * *$ \\
\hline & & & 0,0050 & & & 0,0010 & & & 0,0000 \\
\hline LIP*STOCKTRA & $\begin{array}{c}-0,0042^{* * *} \\
0,0000\end{array}$ & & & & & & & & \\
\hline NIP*STOCKTRA & & $\begin{array}{c}-0,0147^{* * *} \\
0,0000\end{array}$ & & & & & & & \\
\hline TIP*STOCKTRA & & & $\begin{array}{c}-0,0050 * * * \\
0,0000\end{array}$ & & & & & & \\
\hline STOCKTRA & $\begin{array}{c}0,0003^{* * *} \\
0,0000\end{array}$ & $\begin{array}{c}0,0006^{* * *} * \\
0,0000\end{array}$ & $\begin{array}{c}0,0005^{* * *} \\
0,0000\end{array}$ & & & & & & \\
\hline LIP*TUR & & & & $\begin{array}{l}-0,0012 \\
0,2380\end{array}$ & & & & & \\
\hline NIP*TUR & & & & & $\begin{array}{l}-0,0023 \\
0,3380\end{array}$ & & & & \\
\hline TIP*TUR & & & & & & $\begin{array}{c}-0,0016^{*} \\
0,0520\end{array}$ & & & \\
\hline \multirow[t]{2}{*}{ TUR } & & & & $0,0001 * * *$ & $0,0002^{* * *}$ & $0,0002^{* * *}$ & & & \\
\hline & & & & 0,0000 & 0,0000 & 0,0000 & & & \\
\hline LIP*CREDIT & & & & & & & $\begin{array}{c}-0,0026 * * * \\
0,0000\end{array}$ & & \\
\hline NIP*CREDIT & & & & & & & & $\begin{array}{c}-0,0101 * * * \\
0,001\end{array}$ & \\
\hline TIP*CREDIT & & & & & & & & & $\begin{array}{c}-0,0018^{* *} \\
0,0200\end{array}$ \\
\hline \multirow[t]{2}{*}{ CREDIT } & & & & & & & 0,0000 & $0,0001^{*}$ & 0,0000 \\
\hline & & & & & & & 0,9700 & 0,0410 & 0,8290 \\
\hline \multicolumn{10}{|c|}{ Variables de control } \\
\hline \multirow[t]{2}{*}{ INFLA } & $0,0003^{* *}$ & $0,0004^{* *}$ & $0,0004^{* *}$ & $0,0003^{*}$ & $0,0003^{* *}$ & $0,0003 * *$ & $0,0003^{*}$ & $0,0004^{* *}$ & $0,0003^{* *}$ \\
\hline & 0,0160 & 0,0110 & 0,0110 & 0,0540 & 0,0160 & 0,0380 & 0,0700 & 0,0170 & 0,0390 \\
\hline \multirow[t]{2}{*}{ TRADE } & $0,0136 * * *$ & 0,0026 & $0,0077 * * *$ & $0,0106 * * *$ & $0,0103 * * *$ & 0,0052 & $0,0160 * * *$ & $0,0148 * * *$ & $0,0149 * * *$ \\
\hline & 0,0000 & 0,3600 & 0,0010 & 0,0030 & 0,0000 & 0,1980 & 0,0000 & 0,0000 & 0,0000 \\
\hline \multirow[t]{2}{*}{ GCONSUMP } & $-0,0583 * * *$ & $-0,1066 * * *$ & $-0,0769 * * *$ & $-0,0848 * * *$ & $-0,0780 * * *$ & $-0,0985 * * *$ & $-0,0688 * * *$ & $-0,0711 * * *$ & $-0,0724 * * *$ \\
\hline & 0,0000 & 0,0000 & 0,0000 & 0,0000 & 0,0000 & 0,0000 & 0,0000 & 0,0000 & 0,0000 \\
\hline \multirow[t]{2}{*}{ HCAPITAL } & $0,0006^{* * *}$ & $0,0006 * * *$ & $0,0005^{* * *}$ & $0,0006^{* * *}$ & $0,0007 * * *$ & $0,0006 * * *$ & $0,0004^{* * *}$ & $0,0004^{* * *}$ & $0,0004 * * *$ \\
\hline & 0,0000 & 0,0000 & 0,0000 & 0,0000 & 0,0000 & 0,0000 & 0,0000 & 0,0000 & 0,0000 \\
\hline \multicolumn{10}{|l|}{ Períodos } \\
\hline \multirow[t]{2}{*}{$1995-1999$} & 0,0032 & $0,0075^{* *}$ & 0,0005 & $0,0087^{* *}$ & $0,0096 * * *$ & 0,0058 & 0,0047 & 0,0018 & 0,0032 \\
\hline & 0,3180 & 0,0310 & 0,8710 & 0,0110 & 0,0010 & 0,1070 & 0,1230 & 0,4740 & 0,1070 \\
\hline $2000-2004$ & $0,0079 * * *$ & $0,0134 * * *$ & $0,0059 * * *$ & $0,0138 * * *$ & $0,0135 * * *$ & $0,0117^{* * *}$ & 0,0104 & 0,0071 & 0,0098 \\
\hline & 0,0000 & 0,0000 & 0,0000 & 0,0000 & 0,0000 & 0,0000 & 0,0000 & 0,0000 & 0,0000 \\
\hline 2005-2009 & $-0,0076 * * *$ & $0,0077^{* * *}$ & $-0,0072 * * *$ & $0,0054 * * *$ & $0,0064^{* * *}$ & $0,0046 * * *$ & $0,0042^{* * *}$ & $0,0036^{* * *}$ & $0,0039 * * *$ \\
\hline & 0,0000 & 0,0000 & 0,0000 & 0,0000 & 0,0000 & 0,0010 & 0,0000 & 0,0010 & 0,0000 \\
\hline 2010-2014 & $-0,0112 * * *$ & $-0,0026$ & $-0,0103 * * *$ & $-0,0029 *$ & $-0,0056 * * *$ & $-0,0030 *$ & $-0,0043^{*}$ & $-0,0059 * * *$ & $-0,0047 * * *$ \\
\hline & 0,0000 & 0,1190 & 0,0000 & 0,0700 & 0,0000 & 0,0650 & 0,0700 & 0,0000 & 0,0000 \\
\hline Países & 47 & 47 & 47 & 47 & 47 & 47 & 47 & 47 & 47 \\
\hline Observaciones & 229 & 229 & 229 & 228 & 228 & 228 & 230 & 230 & 230 \\
\hline
\end{tabular}

108

Anelena Sabater Castro

(c) (i) (2)

Revista Economía y Sociedad by Universidad Nacional is licensed under a CreativeCommons Reconocimiento-NoComercialCompartirlgual 4.0 Internacional License

Creado a partir de la obra en http://www.revistas.una.ac.cr/index.php/economia 
Economía y Sociedad, Vol. 22, № 52, julio-diciembre 2017, pp. 88-109

EISSN: 2215-3403 URL http://www.revistas.una.ac.cr/economia

\begin{tabular}{|c|c|c|c|c|c|c|c|c|c|}
\hline $\begin{array}{l}\text { Variables } \\
\text { explicativas }\end{array}$ & 1 & 2 & 3 & 4 & 5 & 6 & 7 & 8 & 9 \\
\hline \multicolumn{10}{|c|}{ Pruebas de especificación } \\
\hline $\mathrm{AR}(1)$ & 0,0160 & 0,0150 & 0,0200 & 0,0150 & 0,0170 & 0,0200 & 0,0120 & 0,0230 & 0,0180 \\
\hline $\operatorname{AR}(2)$ & 0,5560 & 0,6830 & 0,6770 & 0,6110 & 0,6970 & 0,7160 & 0,5190 & 0,7420 & 0,6600 \\
\hline \multicolumn{10}{|l|}{ Test de } \\
\hline Hansen & 0,2820 & 0,2240 & 0,3410 & 0,2640 & 0,1510 & 0,3180 & 0,1040 & 0,0860 & 0,1090 \\
\hline
\end{tabular}

Notas: Ver Tabla 1 para consultar las abreviaturas de las variables. $A R(1)$ y $A R(2)$ denota el test ArellanoBond de primer y segundo orden en primeras diferencias. Las estimaciones también incluyen las correspondientes variables dicotómicas de los cinco periodos de tiempo. Variable dependiente: crecimiento real del PIB per cápita. P-values en cursiva. $* * *, * *$ y $*$ denotan significatividad al $1 \%, 5 \%$ y $10 \%$.

Fuente: Elaboración propia. 